Pacific Journal of Mathematics

COMPLETENESS IN SEMIMETRIC SPACE

and Samuel David SHore 


\title{
COMPLETENESS IN SEMIMETRIC SPACES
}

\author{
Fred Galvin and S. D. Shore
}

We establish interrelationships between Cauchy completeness, McAuley's notions of strong and weak completeness, and Moore completeness in semimetrizable spaces as well as developable, 1-continuously, or continuously semimetrizable spaces.

Our main result shows that a semimetrizable space may admit one semimetric which is Cauchy complete and a second semimetric which is developable, and yet will not admit a semimetric which is simultaneously Cauchy complete and developable.

A distance function for a set $X$ is a symmetric, nonnegative real-valued function $d: X \times X \rightarrow \mathbf{R}$ such that $d(x, y)=0$ iff $x=y$. We are interested in the following "continuity properties" often associated with distance functions. A distance function is developable $[\mathbf{P C}]$ iff, for any sequences $\left\langle x_{n}\right\rangle$ and $\left\langle y_{n}\right\rangle$ in $X$ and any $p \in X, \lim d\left(x_{n}, p\right)=\lim d\left(y_{n}, p\right)=0$ implies that $\lim d\left(x_{n}, y_{n}\right)=0$; it is 1-continuous iff, for any $q \in X$, $\lim d\left(x_{n}, p\right)=0$ implies that $\lim d\left(x_{n}, q\right)=d(p, q)$; it is continuous iff $\lim d\left(x_{n}, p\right)=\lim d\left(y_{n}, q\right)=0$ implies that $d\left(x_{n}, y_{n}\right)=d(p, q)$; and it is a metric iff it satisfies the triangle inequality. Obviously, any metric is continuous, and any continuous distance function is both 1-continuous and developable; no other implications hold [B].

A topological space $(X, \mathcal{T})$ is semimetrizable iff there is a distance function $d$ for $X$ such that, for any nonempty subset $A$ of $X$, the closure of $A$ in $(X, \sigma)$ is $\{x \in X \mid d(x, A]=0\}$, where

$$
d(x, A]=\inf \{d(x, a) \mid a \in A\} .
$$

In this case we say that $d$ is an admissible semimetric for $(X, \mathcal{J})$; if, in addition, $d$ is developable, then we say that $d$ is an admissible developable semimetric for $(X, \mathcal{T})$ and that $(X, \mathcal{T})$ is developable semimetrizable. We treat the notions of 1-continuously semimetrizable and continuously semimetrizable similarly.

It is natural to raise the question of whether or not the set of all spheres, $S_{d}(x, \varepsilon)=\{y \in X \mid d(x, y)<\varepsilon\}$, determined by a distance function $d$ is a base for a topology and whether or not this is equivalent to being an admissible semimetric. It is obvious that $d$ is an admissible 
semimetric if and only if, for each $p \in X$, the set $\left\{S_{d}(p, \varepsilon) \mid \varepsilon>0\right\}$ of all spheres centered at $p$ is a neighborhood base for $p$. Hence, if $d$ is an admissible semimetric for $(X, \mathcal{T})$ whose spheres are open, then the set of all spheres is a base for $\mathcal{T}$; the converse may fail. Moreover, when $d$ is an admissible semimetric for a topological space $(X, \mathcal{T})$, there may be spheres that are not open (see, for example, Lemma 2.2); indeed, Heath $\left[\mathbf{H}_{1}\right]$ has a well-known example of a semimetrizable space in which, for any admissible semimetric, its set of spheres is not a base for the topology.

On the other hand, the situation is much simpler if the distance function satisfies a "continuity" condition. For example, if $d$ is a 1-continuous distance function for $X$, then there is a topology $\mathcal{T}$ such that $d$ is an admissible semimetric for $(X, \mathcal{T})$ and the set of all spheres is a base for $\sigma$. Also, a topological space is developable semimetrizable if and only if there is an admissible developable semimetric for the space whose set of spheres is a base for the topology.

Our study focuses on appropriate completeness conditions for semimetrizable spaces and how these interact with the continuity properties.

1. Cauchy completeness versus McAuley completeness. A semimetrizable space $(X, \sigma)$ is Cauchy complete iff it admits a semimetric $d$ such that every $d$-Cauchy sequence converges (where, of course, a sequence $\left\langle x_{n}\right\rangle$ is $d$-Cauchy when, for each $\varepsilon>0$, there is $k$ such that $d\left(x_{n}, x_{m}\right)<\varepsilon$ for any $\left.n, m \geq k\right)$; in this case we say that $d$ is an admissible, Cauchy complete semimetric for $(X, \mathcal{T})$ and that $(X, \mathcal{T})$ is d-Cauchy complete.

This is the first of the completeness concepts that we wish to consider. The study of completeness in semimetric spaces essentially began with two other notions introduced by McAuley in 1956 (see [Mc]).

A semimetrizable space $(X, \mathcal{T})$ is strongly complete iff it admits a semimetric $d$ such that every decreasing sequence $\left\langle F_{n}\right\rangle$ of nonempty closed sets with $F_{n} \subseteq S_{d}\left(x_{n}, 2^{-n}\right)$ for some $x_{n} \in X$ has nonempty intersection; in this case we say that $d$ is an admissible, strongly complete semimetric for $(X, \mathcal{T})$ and that $(X, \mathcal{J})$ is $d$-strongly complete.

Similarly, $(X, \mathcal{T})$ is weakly complete when it admits a semimetric $d$ such that every decreasing sequence $\left\langle F_{n}\right\rangle$ of nonempty closed sets with $F_{n} \subseteq S_{d}\left(x_{n}, 2^{-n}\right)$ for some $x_{n} \in F_{n}$ has nonempty intersection.

The relationships between Cauchy completeness and McAuley's notions of completeness are immediately established, for the most general case, in the following theorem. Our focus is on what more can be said if the distance function has a stronger form of continuity. 
1.1. TheOREM. If $d$ is an admissible semimetric for $(X, \mathcal{T})$, then the following are equivalent:

(1) ( $X, \mathcal{T}$ ) is d-weakly complete;

(2) each $d$-Cauchy filterbase of closed sets converges;

(3) any decreasing sequence $\left\langle F_{n}\right\rangle$ of nonempty closed sets has nonempty intersection, when the diameter of $F_{n}=\sup \left\{d(x, y) \mid x, y \in F_{n}\right\} \leq 2^{-n}$ for each $n$;

(4) each $d$-Cauchy sequence in $X$ has a convergent subsequence.

Proof. The equivalence of (1) and (4) was essentially noted by McAuley [Mc], whereas the other equivalences follow easily from the definitions. Our proof makes use of the well-known fact that $d$ is admissible for $(X, \mathcal{T})$ if and only if $\mathscr{T}$ is first countable and a sequence $\left\langle x_{n}\right\rangle$ converges to $p$ iff $d\left(x_{n}, p\right) \rightarrow 0$.

1.2. REMARK. It is now obvious that, if $(X, \mathcal{T})$ is $d$-strongly complete or $d$-Cauchy complete, then $(X, \mathcal{T})$ is $d$-weakly complete. The following remarks show that the converse fails.

1.3. Remark. A Cauchy complete space need not be strongly complete.

Consider the Niemytzki space $\Gamma$ (see [GJ; 3K]). If the distance between points is the diameter of the smallest closed disc in the upper half plane that contains the two points (with 1 as the maximum distance between any two points), then this is an admissible continuous semimetric for $\Gamma$ such that every Cauchy sequence converges (see $[\mathbf{K}]$ ).

On the other hand, Heath $\left[\mathbf{H}_{3}\right]$ has shown that any regular, separable, strongly complete semimetrizable space is metrizable. Since $\Gamma$ is not metrizable, it follows that $\Gamma$ is not strongly complete.

Notice that this example also shows that a continuously semimetrizable, weakly complete space need not be strongly complete.

1.4. RemarK. A topological space may admit a strongly complete developable semimetric that is not Cauchy complete.

First, note that, if $(X, \mathcal{T})$ is countably compact, then every admissible semimetric for $(X, \mathcal{T})$ is strongly complete. Moveover, if $(X, \mathcal{T})$ is developable semimetrizable and $\left\langle x_{n}\right\rangle$ is any sequence of distinct points in $X$, then there is an admissible, developable semimetric $d$ for $(X, \mathcal{T})$ such that $\left\langle x_{n}\right\rangle$ is a $d$-Cauchy sequence; hence, if $(X, \mathcal{T})$ is a developable semimetrizable space with a divergent sequence of distinct points, then $(X, \widetilde{T})$ admits a developable semimetric that is not Cauchy complete. 
Now, let $(X, \mathcal{T})$ be any compact metrizable topological space with at least two non-isolated points. Then, $(X, \mathcal{T})$ does admit a developable semimetric (and, therefore, a strongly complete one) that is not Cauchy complete. For example, let $X$ be the subspace $A \cup B \cup\{0,1\}$ of the space of real numbers with the usual topology, where $A$ is the set of all numbers $2^{-n}$ and $B$ is the set of all numbers $1+3^{-n}$ for any positive integer $n$. There is an admissible developable semimetric for $(X, \mathcal{T})$ such that $d(x, y)=|x-y+1|$, if $x \in A$ and $y \in B$, and, otherwise, $d(x, y)=$ $|x-y|$; however, there is a $d$-Cauchy sequence that fails to converge (e.g., $x_{2 n}=2^{-2 n}$ and, otherwise, $x_{n}=1+3^{-n}$ ).

Finally, stronger continuity assumptions can produce stronger completeness interrelationships.

1.5. THEOREM. For any 1-continuous semimetric $d,(X, \mathcal{T})$ is $d$-Cauchy complete if and only if it is d-weakly complete.

Proof. Let $d$ be an admissible 1-continuous semimetric for $(X, \mathcal{T})$ and $\left\langle x_{n}\right\rangle$ be a $d$-Cauchy sequence that has a subsequence $\left\langle x \circ k_{n}\right\rangle$ which converges to $p$; it follows that

$$
\lim _{n} d\left(x_{n}, p\right)=\lim _{n} \lim _{m} d\left(x_{n}, x \circ k_{m}\right)=0 .
$$

That is, if $d$ is 1-continuous, then any $d$-Cauchy sequence with a convergent subsequence is convergent. Therefore, if $(X, \mathcal{T})$ is $d$-weakly complete, then $(X, \mathcal{T})$ is $d$-Cauchy complete; the converse always holds.

2. Cauchy complete and developable semimetrics. Our results of the preceding section were obtained in answer to questions of how Cauchy, strong, and weak completeness interrelate and of what more can be said in the presence of stronger continuity properties. A question that follows easily in this context is this: Suppose that a semimetrizable space admits two semimetrics, one that has a strong continuity property and another that has a nice completeness property. Is there an admissible semimetric for the space that has both the continuity property and the completeness property?

We have solved this problem (in the negative) for the case of Cauchy complete and developable semimetrics with the following theorem.

2.1. Theorem. A semimetrizable space may be Cauchy complete and developable and yet admit no semimetric which is (simultaneously) Cauchy complete and developable. 
We claim that the Isbell-Mrówka spaces $\psi_{\Re}$ provide such an example.

Recall that, for any family $R$ of infinite, almost disjoint subsets of $\mathbf{N}$ (the set of natural numbers), there is a topology for $\mathbf{N} \cup \Re$ such that each $n \in \mathbf{N}$ is isolated and each $A \in \Re$ has a countable local base, consisting of sets of the form $\{A\} \cup\{m \in A \mid m \geq n\}$ for each $n \in \mathbf{N}$. (In this case a family is almost disjoint if the intersection of any two distinct members is finite.) Following [GJ; 5I], we denote this space by $\psi_{\Re}$.

It is well known that these spaces are completely regular, Hausdorff, but not normal (and, therefore, not metrizable), when the family $\Re$ is infinite and maximal. For more information concerning these spaces, we refer the reader to [GJ].

2.2. LEMMA. For any family $\mathcal{R}$, there is an admissible Cauchy complete semimetric for $\psi_{\Omega}$.

Proof. There is a distance function $d_{1}$ for $\mathbf{N} \cup \Re$ such that, for $x \neq y$,

$$
d_{1}(x, y)=d_{1}(y, x)= \begin{cases}x^{-1}, & \text { if } x \in y \in \Re \\ 1, & \text { otherwise. }\end{cases}
$$

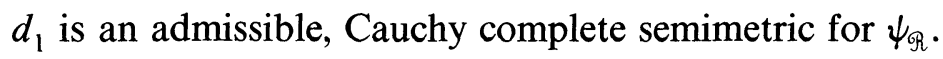

2.3. LEMMA. For any infinite, maximal family $\Re$, there is an admissible developable weakly complete semimetric for $\psi_{\mathcal{R}}$ that is not Cauchy complete.

Proof. There is a distance function $d_{2}$ for $\mathbf{N} \cup \Re$ such that, for $x \neq y$,

$$
d_{2}(x, y)=d_{2}(y, x)= \begin{cases}x^{-1}, & \text { if } x \in y \in \Re \\ \left|x^{-1}-y^{-1}\right|, & \text { if } x, y \in \mathbf{N} \\ 1, & \text { otherwise }\end{cases}
$$

$d_{2}$ is an admissible, developable semimetric for $\psi_{\Re}$ such that any $d_{2}$-Cauchy sequence has a convergent subsequence (i.e., $d_{2}$ is weakly complete). But, not every $d_{2}$-Cauchy sequence converges; in particular, $\langle n\rangle$ is a $d_{2}$-Cauchy sequence that does not converge.

2.4. REMARK. By using an argument similar to that in Remark 1.3, it follows that $\psi_{\Re}$ is not strongly complete when $R$ is an infinite, maximal almost disjoint family. From this and Lemma 2.3 we conclude that the converse of Remark 1.2 may fail; that is, even for developable distance functions $d,(X, \mathcal{T})$ may be $d$-weakly complete without being either $d$-Cauchy complete or $d$-strongly complete. 
2.5. REMARK. We intend to show that, for any maximal almost disjoint family $\Re, \psi_{\Re}$ does not admit a developable, Cauchy complete semimetric. But first, we give an easy cardinality argument which shows that, for most choices of $\Re$, there is no admissible, developable, Cauchy complete semimetric for $\psi_{\Re}$.

Let us call two subsets of $\mathbf{N}$ equivalent if their symmetric difference is finite, and two maximal almost disjoint families $R$ and $R *$ equivalent if $\Re^{*}$ may be obtained from $\Re$ by replacing each $A \in \Re$ by a set $A^{*}$ that is equivalent to $A$. Note that, if $d$ is an admissible developable semimetric for $(X, \mathcal{T})$, then each convergent sequence is $d$-Cauchy. Hence, if $d$ is a developable and Cauchy complete semimetric for $\psi_{\Re}$, then the family $R$ is determined (up to equivalence) by the values $d$ takes on $\mathbf{N} \times \mathbf{N}$. Now, there are only $2^{\aleph_{0}}$ such semimetrics on $\mathbf{N}$, while there are $2^{2^{N_{0}}}$ inequivalent maximal almost disjoint families of subsets of $\mathbf{N}$. Consequently, most of the spaces $\psi_{\Re}$ do not admit Cauchy complete developable semimetrics.

2.6. LEMMA. For any infinite, maximal family $\Re$, there is no admissible semimetric for $\psi_{\mathcal{R}}$ which is both Cauchy complete and developable.

Proof. Suppose, on the contrary, that $d$ is an admissible Cauchy complete and devlopable semimetric for $\psi_{\mathcal{R}}$ where $\Re$ is maximal and infinite.

We may assume that the range of $d$ is contained in $\{0\} \cup\left\{n^{-1} \mid n \in\right.$ $\mathbf{N}$; ; otherwise, replace $d$ by an equivalent semimetric $d^{*}$ with, for $x \neq y$,

$$
d^{*}(x, y)=\left(\left[\frac{1}{d(x, y)}\right]+1\right)^{-1}
$$

Therefore, if $\left\langle x_{n}\right\rangle$ is not a $d$-Cauchy sequence, then there is a subsequence $\left\langle x_{k_{n}}\right\rangle$ such that $d\left(x_{k_{2 m-1}}, x_{k_{2 m}}\right)=d\left(x_{k_{2 m+1}}, x_{k_{2 m+2}}\right)$ for all $m$.

Now, for each $i \in \mathbf{N}$, choose distinct $A_{i} \in \mathcal{R}$ and define $A_{i}^{*}=$ $A_{i} \backslash \cup_{j=1}^{i-1} A_{j}$. Thus, we obtain the pairwise disjoint family $\left\{A_{i}^{*} \mid i \in \mathbf{N}\right\}$. Let $\left\{a_{\imath} \mid j \in \mathbf{N}\right\}$ be an enumeration of $A_{i}^{*}$ without repetition.

For any set $S,[S]^{8}$ is the set of all 8-element subsets of $S$.

Define a partition of [N] $]^{8}$ into two sets $J$ and $K$ as follows: $X \in J$ iff $d\left(a_{i_{1} l_{2}}, a_{i_{3} i_{4}}\right)=d\left(a_{i_{5} i_{6}}, a_{i_{7} i_{8}}\right)$, where $X=\left\{i_{1}, i_{2}, i_{3}, i_{4}, i_{5}, i_{6}, i_{7}, i_{8}\right\}$ is an increasing listing of $X$.

It follows from Ramsey's Theorem [Ra; Theorem A] that there is an infinite set $M \subseteq \mathbf{N}$ such that $[M]^{8} \subseteq J$ or $[M]^{8} \subseteq K$. We intend to show that neither alternative is possible and, thereby, arrive at a contradiction which will establish Lemma 2.6. 
First, $[M]^{8} \subseteq J$ is impossible. Otherwise, let $\left\{i_{n} \mid n \in \mathbf{N}\right\}$ be an increasing enumeration of $M$. The maximality of $\Re$ insures that any sequence of distinct elements in $\mathbf{N}$ has a convergent subsequence. However, the sequence $\left\langle a_{i_{2 n-1} i_{2 n}}\right\rangle$ has no $d$-Cauchy subsequences and, therefore, no convergent subsequences (since $d$ is developable).

But also, $[M]^{8} \subseteq K$ is impossible. Otherwise, for any increasing sequence $\left\langle i_{n}\right\rangle$ in $M$, the sequence $\left\langle a_{i_{2 n-1} l_{2 n}}\right\rangle$ is $d$-Cauchy and, therefore, convergent. We shall refer to sequences of this form as $M$-sequences.

Now, note that all $M$-sequences converge to the same point in $R$. (To see this, observe that, for any two $M$-sequences, there is a third $M$-sequence which has a subsequence in common with each of the first two.) Let $B$ denote the (common) limit of all $M$-sequences; since no $M$-sequence can converge to any of the points $A_{l}$, we conclude that $B \neq A_{i}$ for each $i \in \mathbf{N}$.

Since $\Re$ is almost disjoint, for each $i \in \mathbf{N}$ we have $a_{l j} \notin B$ for all sufficently large $j$. Hence, we can find an increasing sequence $\left\langle i_{n}\right\rangle$ in $M$ such that $a_{t_{2 n-1} l_{2 n}} \notin B$ for all $n$. Since $\left\langle a_{l_{2 n-1} l_{2 n}}\right\rangle$ is an $M$-sequence and does not converge to $B$, we have a contradiction.

Lemmas 2.2, 2.3 and 2.6 establish a proof for Theorem 2.1.

2.7. REMARK. J. Roitman has pointed out that a proof of Lemma 2.6 also follows from some results of A. R. D. Mathias.

Let $\mathscr{G}$ be the ideal in $\mathscr{P}(\mathbf{N})$, the Boolean algebra of subsets of $\mathbf{N}$, that is generated by the maximal almost disjoint family $R$ along with the finite subsets of $\mathbf{N}$. According to Mathias [M;0.7, 4.6], $\mathscr{P}(\mathbf{N}) \backslash \mathscr{G}$ cannot be analytic.

However, if $d$ is an admissible Cauchy complete developable semimetric for $\psi_{\Re}$, we have that $X \in(\mathscr{P}(\mathbf{N}) \backslash \mathscr{G})$ if and only if there are infinitely many $d$-Cauchy sequences of distinct points in $X$ such that the "union" of no two of them is $d$-Cauchy.

Now, let $B$ be the set of all points $(x, y) \in 2^{\mathbf{N}} \times \mathbf{N}^{\mathbf{N} \times \mathbf{N}}$ such that, for $i, j, m, n \in \mathbf{N}$, (1) $\left\{y_{l j} \mid i, j \in \mathbf{N}\right\} \subseteq\left\{n \mid x_{n}=1\right\}$; (2) If $m \neq n$, then $y_{i m} \neq y_{i n} ;(3)\left\langle y_{i n}\right\rangle$ is a $d$-Cauchy sequence; and (4) if $i \neq j$, then $\left\langle y_{i 1}, y_{j 1}\right.$, $\left.y_{t 2}, y_{j 2}, \ldots\right\rangle$ is not a $d$-Cauchy sequence. Note that $B$ is a Borel set (in fact, an $\left.F_{\sigma \delta \sigma \delta}\right)$. If we identify $\mathscr{P}(\mathbf{N})$ with $2^{\mathbf{N}}$ in the usual way, then $2^{\mathbf{N}} \backslash \mathscr{9}$ is precisely the projection of $B$ (in the first coordinate). Thus, $\mathscr{P}(\mathbf{N}) \backslash \mathscr{G}$ is the projection of a Borel set (i.e., analytic) which, of course, contradicts the results of Mathias.

3. Concerning developments. A topological space $(X, \mathcal{T})$ is developable in the sense of $R$. L. Moore iff it is a $T_{1}$-space for which there is a 
sequence $\left\langle\mathcal{G}_{n}\right\rangle$ of open covers of $X$ (called a development) such that $\mathcal{G}_{n+1} \subseteq \mathcal{G}_{n}$ for each $n$ and, for each $p \in X,\left\{\operatorname{st}\left(p, \mathcal{G}_{n}\right) \mid n \in \mathbf{N}\right\}$ is a local base for $p$, where st $\left(p, \mathcal{G}_{n}\right)=\cup\left\{G \mid p \in G \in \mathcal{G}_{n}\right\}$; in this case we say that $(X, \mathcal{J})$ admits the development $\left\langle\mathcal{G}_{n}\right\rangle$ or that $\left\langle\mathcal{G}_{n}\right\rangle$ is a development for $(X, \mathcal{T})$. (The previous use of developable semimetrizable is, of course, intentional; see Theorem 3.1 below.)

A Moore space is a regular Hausdorff space that admits a development.

A development for a topological space $(X, \mathcal{T})$ is complete when any decreasing sequence $\left\langle F_{n}\right\rangle$ of nonempty closed sets such that $F_{n} \subseteq G_{n}$ for some $G_{n} \in \mathcal{G}_{n}$ has nonempty intersection. We address the question of how this notion of completeness relates to the (distance function) notions of completeness that we have considered previously.

Again, our results center around developable semimetrics. In this case the useful known results are these. A distance function for $X$ is developable if and only if there are spheres of arbitrarily small diameter centered at each point of $X$. Hence, if $d$ is an admissible developable semimetric for $(X, \mathcal{T})$, then the set $\delta_{n}$ of all open sets of diameter less than or equal to $2^{-n}$ is an open cover of $X$ so that $\left\langle\delta_{n}\right\rangle$ is a development for $(X, \mathcal{T})$.

On the other hand, if $\left\langle\mathcal{G}_{n}\right\rangle$ is a development for $(X, \mathcal{T})$, then there is a distance function $d$ for $X$ such that $d(x, y)=2^{-n}$, where $n$ is the first integer such that $x \notin \operatorname{st}\left(y, \mathcal{G}_{n}\right)$. It follows that $d$ is an admissible developable semimetric for $(X, \mathcal{T})$ with $S_{d}\left(p, 2^{-n}\right)=\operatorname{st}\left(p, \mathcal{G}_{n}\right)$.

These standard techniques (see, for example, [Mc], [Re], [S], and references therein) for relating distance functions and developments lead directly to proofs for each of the following theorems.

3.1. TheOREM. A topological space $(X, \mathcal{T})$ is developable semimetrizable if and only if it is developable in the sense of $R . L$. Moore.

3.2. ThEOREM. If $(X, \mathcal{T})$ admits a weakly complete, developable semimetric, then there is a complete development for $(X, \sigma)$.

3.3. THEOREM. ( $X, \mathcal{T})$ admits a strongly complete, developable semimetric if and only if $(X, \mathcal{T})$ is sequentially complete (i.e., it is a $T_{1}$-space for which there is a development $\left\langle\mathcal{G}_{n}\right\rangle$ such that every decreasing sequence $\left\langle F_{n}\right\rangle$ of nonempty closed sets with $F_{n} \subseteq \operatorname{st}\left(x_{n}, \mathcal{G}_{n}\right)$ for some $x_{n} \in X$ has nonempty intersection).

3.4. REMARK. Our Theorem 3.3 clarifies and generalizes several results in [Re; see Lemmas 1 and 2 in particular], where sequentially complete is defined and attributed to D. R. Traylor. 
There is, of course, an analogous theorem to 3.3 for spaces that admit weakly complete, developable semimetrics.

\section{REFERENCES}

[B] Carlos J. R. Borges, On continuously semimetrizable and stratifiable spaces, Proc. Amer. Math. Soc., 24 (1970), 193-196.

[GJ] L. Gillman and M. Jerison, Rings of Continuous Functions, D. Van Nostrand \& Co., Princeton, N.J., 1960.

$\left[\mathrm{H}_{1}\right] \quad$ Robert W. Heath, A regular semi-metric space for which there is no semi-metric under which all spheres are open, Proc. Amer. Math. Soc., 12 (1961), 810-811.

$\left[\mathrm{H}_{2}\right] \ldots$, Arc-wise connectedness in semi-metric spaces, Pacific J. Math., 12 (1962), 1301-1319.

$\left[\mathrm{H}_{3}\right] \longrightarrow$ Separability and $\boldsymbol{\aleph}_{1}$-compactness, Colloq. Math., 12 (1964), 11-14.

[K] Steven A. Kenton, Neometric Spaces, Dissertation, University of New Hampshire, 1971.

[M] A. R. D. Mathias, Happy families, Ann. Math. Logic, 12 (1977), 59-111.

[Mc] Louis F. McAuley, A relation between perfect separability, completeness, and normality in semi-metric spaces, Pacific J. Math., 6 (1956), 315-326.

[PC] A. D. Pitcher and E. W. Chittenden, On the foundations of the calcul fonctionnel of Fréchet, Trans. Amer. Math. Soc., 19 (1918), 66-78.

[Ra] Frank P. Ramsey, On a problem of formal logic, Proc. London Math. Soc., (2) 30 (1930), 264-286.

[Re] G. M. Reed, On metrizability of complete Moore spaces, Pacific J. Math., 50 (1974), 595-599.

[S] S. D. Shore, Coherent distance functions, Topology Proceedings, 6 (1981), 405-422.

Received April 12, 1982 and in revised form May 12, 1983. The first author received support from NSF grant MCS 77-02046 A01.

UNIVERSITY OF KANSAS

LAWRENCE, KS 66045

AND

UNIVERSITY OF NEW HAMPSHIRE

DURHAM, NH 03824 



\section{PACIFIC JOURNAL OF MATHEMATICS \\ EDITORS}

Donald BABBITT (Managing Editor)

University of California

Los Angeles, CA 90024

Hugo Rossi

University of Utah

Salt Lake City, UT 84112

C. C. Moore and Arthur Ogus

University of California

Berkeley, CA 94720
J. DugunduI

Department of Mathematics

University of Southern California

Los Angeles, CA 90089-1113

R. FinN and H. SAMELSON

Stanford University

Stanford, CA 94305

ASSOCIATE EDITORS

R. ARENS

E. F. BECKENBACH

B. H. NeUMANN

F. WOLF

K. YosHIDA (1906-1982)

\section{SUPPORTING INSTITUTIONS}

UNIVERSITY OF ARIZONA

UNIVERSITY OF BRITISH COLUMBIA

CALIFORNIA INSTITUTE OF TECHNOLOGY

UNIVERSITY OF CALIFORNIA

MONTANA STATE UNIVERSITY

UNIVERSITY OF NEVADA, RENO

NEW MEXICO STATE UNIVERSITY

OREGON STATE UNIVERSITY
UNIVERSITY OF OREGON

UNIVERSITY OF SOUTHERN CALIFORNIA

STANFORD UNIVERSITY

UNIVERSITY OF HAWAII

UNIVERSITY OF TOKYO

UNIVERSITY OF UTAH

WASHINGTON STATE UNIVERSITY

UNIVERSITY OF WASHINGTON 


\section{Pacific Journal of Mathematics}

\section{Vol. 113, No. $1 \quad$ March, 1984}

Flavio E. A. da Silveira, Rational homotopy theory of fibrations $\ldots \ldots \ldots \ldots 1$

Donald M. Davis, Desuspensions of stunted projective spaces ............ 35

Lou van den Dries, Exponential rings, exponential polynomials and

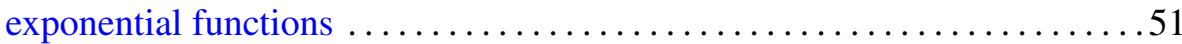

Fred Galvin and Samuel David Shore, Completeness in semimetric

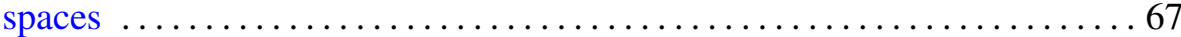

Fereidoun Ghahramani, Compact elements of weighted group algebras . . . 777

Munehiko Itōo, The closed image of a hereditary $M_{1}$-space is $M_{1} \ldots \ldots \ldots 85$

Elvira Laura Livorni, Classification of algebraic surfaces with sectional genus less than or equal to six. I. Rational surfaces .............. 93

H. Alan MacLean, Riesz sets and a theorem of Bochner ............ 115

E. Neher, Jordan triple systems with completely reducible derivation or

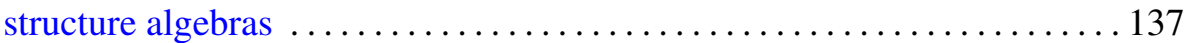

Joe Repka, Shalika's germs for $p$-adic GL( $n)$. I. The leading term $\ldots \ldots \ldots 165$

Joe Repka, Shalika's germs for $p$-adic GL( $n)$. II. The subregular term $\ldots \ldots 173$

Rae Michael Andrew Shortt, Borel density, the marginal problem and isomorphism types of analytic sets $\ldots \ldots \ldots \ldots \ldots \ldots \ldots \ldots \ldots \ldots \ldots \ldots \ldots$

Baruch Solel, The multiplicity functions of invariant subspaces for nonselfadjoint crossed products $\ldots \ldots \ldots \ldots \ldots \ldots \ldots \ldots \ldots \ldots \ldots \ldots . \ldots \ldots$

Su-win Yang, Self-intersection number of immersions and enumeration of nonstable vector bundles

W. M. Zajączkowski, Local solvability of nonstationary leakage problem for ideal incompressible fluid. II 